\title{
Solution structures of the Shewanella woodyi H-NOX protein in the presence and absence of soluble guanylyl cyclase stimulator IWP-051
}

\author{
Cheng-Yu Chen ${ }^{1}$, Woonghee Lee ${ }^{2,3}$ and William R. Montfort ${ }^{1 *}$
}

${ }^{1}$ Department of Chemistry and Biochemistry, University of Arizona, Tucson, AZ, 85721

${ }^{2}$ National Magnetic Resonance Facility at Madison, Biochemistry Department, University of Wisconsin-Madison, Madison, WI 53706, USA.

${ }^{3}$ Department of Chemistry, University of Colorado Denver, Denver, CO 80238, USA

*Correspondence: montfort@arizona.edu.

Running head: Solution structure of the S. woodyi H-NOX protein 


\section{Abstract}

Heme-nitric oxide/oxygen binding (H-NOX) domains bind gaseous ligands for signal transduction in organisms spanning prokaryotic and eukaryotic kingdoms. In the bioluminescent marine bacterium Shewanella woodyi $(S w)$, H-NOX proteins regulate quorum sensing and biofilm formation. In higher animals, soluble guanylyl cyclase (sGC) binds nitric oxide with an H-NOX domain to induce cyclase activity and regulate vascular tone, wound healing and memory formation. SGC also binds stimulator compounds targeting cardiovascular disease. The molecular details of stimulator binding to SGC remain obscure but involve a binding pocket near an interface between $\mathrm{H}-\mathrm{NOX}$ and coiled-coil domains. Here, we report the full NMR structure for CO-ligated Sw H-NOX in the presence and absence of stimulator compound IWP-051, and its backbone dynamics. Non-planar heme geometry was retained using a semi-empirical quantum potential energy approach. Although IWP-051 binding is weak, a single binding conformation was found at the interface of the two H-NOX subdomains. Binding lead to rotation of the subdomains and closure of the binding pocket. Backbone dynamics for the protein are similar across both domains except for two helix-connecting loops, which display increased dynamics that are further enhanced by compound binding. Structure-based sequence analyses indicate high sequence diversity in the binding pocket, but the pocket itself appears conserved among H-NOX proteins. The largest dynamical loop lies at the interface between Sw H-NOX and its binding partner as well as in the interface with the coiled coil in SGC, suggesting a critical role for the loop in signal transduction.

Keywords: nitric oxide | drug binding | NMR | solution structure | H-NOX | guanylate cyclase

Abbreviations used: AUDANA, Automated Database-Assisted NOE Assignment; BMRB, Biological Magnetic Resonance Bank; cryo-EM, cryogenic electron microscopy; CSP, chemical shift perturbation; Cb, Clostridium botulinum; Cs, Caldanaerobacter subterraneus; hetNOE, 
heteronuclear NOE; H-NOX, heme-nitric oxide/oxygen binding; HSQC, heteronuclear single quantum coherence; Ka Kordia algicida; Ms Manduca sexta; PDB, Protein Data Bank; PONDEROSA, Peak-picking Of NOE Data Enabled by Restriction of Shift Assignments; NMR, Nuclear Magnetic Resonance; NMRFAM, National Magnetic Resonance Facility at Madison; NO, nitric oxide; NOE, nuclear Overhauser effect; NOESY, nuclear Overhauser enhancement spectroscopy; Ns, Nostoc sp. PCC 7120; PAH, pulmonary arterial hypertension; PAS, Per-ARNTSim; RDC, residual dipolar coupling; RMSD, root-mean-square deviation; sGC, soluble guanylyl cyclase; So Shewanella oneidensi; TrNOESY, transferred NOESY; TROSY, transverse relaxation optimized spectroscopy;

(Graphical abstract and Highlights included separately) 


\section{Introduction}

H-NOX domains are gas-sensing hemoproteins that bind dissolved gases and induce signaling responses in a wide variety of organisms. In prokaryotes, H-NOX sensors function as both stand-alone proteins and as members of multidomain proteins, and respond to nitric oxide, oxygen or potentially other ligands to induce a signaling cascade [1, 2]. Obligate anaerobes use $\mathrm{H}-\mathrm{NOX}$ domains to escape dioxygen as part of a methyl-accepting chemotaxis system while a variety of bacteria use stand-alone H-NOX proteins to sense nitric oxide and repress biofilm formation through lowering cyclic di-GMP levels. In the latter case, H-NOX proteins may participate in a two-component signaling cascade, inhibiting a histidine kinase that stimulates a cyclic di-GMP synthase, or by directly stimulating the phosphodiesterase activity, and inhibiting the cyclase activity, of a cyclic di-GMP synthase/phosphodiesterase fusion protein. The H-NOX protein from Shewanella woodyi (Sw H-NOX) is a member of this last category.

In animals, H-NOX domains are part of SGC proteins that generally sense nitric oxide (NO) but may also sense oxygen, for example in the worm $C$. elegans where oxygen levels regulate foraging $[3,4]$. Best studied are the heterodimeric sGC proteins from insects and mammals. Heterodimeric sGC is composed of two chains, $\alpha$ and $\beta$, produced through gene duplication with each chain containing an N-terminal H-NOX domain, a central PAS (Per-ARNT-Sim) domain, a coiled-coil domain and a C-terminal cyclase domain $[5,6]$. The $\alpha \mathrm{H}-\mathrm{NOX}$ domain has lost the ability to bind heme and is best categorized as a pseudo H-NOX domain. By producing cGMP in response to NO binding, heterodimeric SGC regulates numerous physiological processes in animals, including blood pressure, wound healing and memory formation, and is also emerging as antitumorigenic.

Loss of cGMP production through impaired sGC is linked to hypertension, atherosclerosis, asthma, neurodegeneration and contributes to heart attack and stroke. New sGC stimulating compounds, which act alone and in synergy with NO binding, are showing clinical promise and 
one such compound, riociguat (trade name Adempas), is in clinical use for pulmonary arterial hypertension (PAH) and related disorders [7]. sGC stimulators all derive from YC-1 (Figure 1), which was initially discovered as an antiplatelet compound [8] and eventually shown to target sGC. More recently, compound IWP-051 was developed [9], the precursor to praliciguat, which is now in clinical trial for treating hypertension in diabetics, including those with diabetic nephropathy [7].

Despite the promise of SGC stimulators, where they bind and how they work remains unclear. The H-NOX domain from Clostridium botulinum, also referred to as sensor of nitric oxide ( $C b$ SONO), was reported to have altered CO release kinetics in the presence of SGC stimulator BAY 41-2272, suggesting binding was to the NO-sensing domain in SGC [10]. We recently found that a photoactivatable version of IWP-051 covalently attaches to residues in the $\beta \mathrm{H}$-NOX domain and in the coiled-coil domain [11], consistent with previous studies indicating the H-NOX domain was in contact with the coiled-coil domain $[12,13]$. We also showed weak binding to H-NOX proteins Nostoc sp. PCC 7120 (Ns H-NOX), Shewanella oneidensis (So H-NOX), Shewanella woodyi (Sw H-NOX) and Cb SONO. More recently, moderate resolution $(3.8-5.8 \AA)$ models for sGC in inactive and active conformations were published, showing SGC to undergo a dramatic change in conformation upon activation that involves kink-straightening and repacking of the coiled-coil domain [14, 15], which has conserved positions of instability to facilitate activation [16]. The H-NOX domain lies along the coiled coil in these structures, and in one, density for YC-1 can be seen at the interface of the H-NOX and coiled-coil domains [15].

Here, we describe the full NMR structures of Sw H-NOX in the absence and presence of stimulator IWP-051, which reveals a conserved pocket in the protein. We also describe the backbone dynamics for Sw H-NOX and how it changes in response to IWP-051 binding. 


\section{Results}

\section{NMR structure determination of the Sw H-NOX CO complex.}

Sw H-NOX was expressed in E. coli, purified with fully intact heme as a ferric/ferrous mixture and reduced to the ferrous state using dithionite, as previously described [11]. The protein contains the full protein (182 residues) plus eight additional residues at the C-terminus arising from the purification tag after cleavage with TEV protease. For NMR studies, we focused on the $\mathrm{Fe}(\mathrm{II})-\mathrm{CO}$ complex, which is diamagnetic and best suited for analysis [17]. The Fe(II)-CO Sw HNOX complex yielded a well-dispersed ${ }^{1} \mathrm{H}-{ }^{15} \mathrm{~N}$ HSQC spectrum that was consistent with previous studies [11, 18] and unchanged for at least one week at room temperature, indicating high complex stability (Figure S1). Chemical shift assignments for Sw H-NOX were obtained using standard triple-resonance and NOESY experiments (described in Supplementary Information). Approximately $97 \%$ of backbone resonances and $80 \%$ of side chain resonances were assigned. The majority of unassigned resonances appear to be in the flexible loops of residues $30-45$ and 81-90.

The Fe(II)-CO complex structure was determined using Xplor-NIH [19] and the following input data: NOE-derived distance restraints calibrated by NMRFAM-SPARKY [20], chemical shiftderived dihedral angle restraints from Talos- $\mathrm{N}$ prediction [21], hydrogen bonding restraints generated with the AUDANA algorithm [22], and global orientation restraints from residual dipolar couplings (RDCs). The AUDANA algorithm generated idealized $\mathrm{H}$-bond restraints from NOE patterns and the secondary structures predicted by Talos-N. The restraints were cross evaluated with the NOESY spectrum and the structures generated during each cycle of structure calculations. Violations to the NOESY spectrum were removed during each cycle. We chose the least energy structure from 200 calculated structures for further complex structure calculation steps.

Modeling heme by NMR in heme-containing proteins is challenging due to the sparse number of NOE restraints available. To begin, we modeled Sw H-NOX without heme, located the 
heme pocket and added heme. Heme-protein NOEs guided heme placement and were consistent with the expected heme pocket from crystal structures of homologous proteins. However, the apo-model refined with a too-small heme pocket and heme-docking resulted in numerous steric clashes. To reconstitute a proper heme binding pocket, 27 loose long-range pseudo CA-CA NOE distance restraints with high lower limit boundaries were introduced for residues surrounding the heme binding pocket, which led to improved overall structure quality, improved RDC correlation ( 0.98 to $\sim 0.995)$ and no increase in NOE violations. These longrange restraints were removed once heme was reliably placed in the model.

Model refinement was guided by strong NOEs between the heme methine protons and protein side chains including residues L78, I79, L95, I99, I103, V107, A114, L116, A143, and L146. The Fe-NE2 distance between heme and His 104, the proximal histidine, was restrained to $2.1 \pm 0.1 \AA$ and the distance between the porphyrin ring nitrogen and His 104 NE2 restrained to $3.0 \pm 0.1 \AA$. The Fe-CO bond was fixed at $1.9 \AA$ and the Fe-C-O bond angle at $180^{\circ}$, as expected for Fe(II)-CO [23-25]. Heme methyl and vinyl groups were restrained by intra-heme NOEs derived from a 2D-[F1,F2]- ${ }^{13} \mathrm{C}^{15} \mathrm{~N}$-filtered NOESY experiment (Figure S2). The heme conformation was further refined with addition of heme-protein NOEs (109 in all, Table S1), which indicated a single heme orientation was present $[26,27]$. NOEs were not observed for the second of the two possible heme orientations, one flipped with respect to the other, unlike in, for example, nitrophorin 4, where the two possible heme orientations exist in roughly equal proportion [27, 28]. A lack of unambiguous NOE restraints led to high flexibility in the heme propionates during docking, and steric clashes with protein loop residues 113-117, 133-138 and the N-terminal amine. H-NOX family proteins have a conserved $Y x S x R$ motif that hydrogen bonds to the propionate groups [29] and we therefore added a restraint between these residues (Y133, S135 and $\mathrm{R} 137)$ and the propionate oxygens $(5.0 \AA)$. The N-terminal amine was additionally restrained to be near a heme propionate but with greater variability allowed $(4.5 \pm 0.5 \AA)$. Including these 
restraints reduced steric clashes without generating NOE violations or changing backbone structure.

Heme geometry is also difficult to model by NMR, again due to the paucity of NMR restraints and the flexibility of heme. H-NOX proteins have non-planar heme geometries that vary in magnitude among the different members of the H-NOX family, adding additional complexity to modeling efforts [29-31]. To preserve heme non-planarity, we calculated topology and energy parameters optimized for non-planar heme using the semi-empirical quantum chemistry potential energy approach of MOPAC PM7, which was designed using experimental and ab initio reference data for biological systems [32]. During docking, the heme potential energy for bonds and angles was scaled to the protein potential energy (with satisfied NMR restraints), while the potential energy for heme dihedral and improper angles was left unchanged, allowing the heme to sample nonplanar conformations.

The resulting models were refined using the EEFx2 potential energy function, which simultaneously implements solvation, electrostatics, and van der Waals energy terms, allowing for a more physically realistic calculation [33]. The EEFx2 force field with implicit water was incorporated into the final structure refinement [33]. To represent the solution structure ensemble, 20 models with low energy and best data agreement (no NOE violations $>0.5 \AA$ and no dihedral angle violations $>5^{\circ}$ ) were chosen from 200 random starting models (Figure 2a). A summary of structure statistics is provided in Table S1.

The final Sw H-NOX models displayed overall fold and heme conformations that are similar to those found in other $\mathrm{H}-\mathrm{NOX}$ structures, including the $\mathrm{CO}$ complexes from Nostoc sp. PCC 7120 (PDB ID 2O0G [31]), Shewanella oneidensis (PDB IDs 2KII [34] and 4U9G [35]), and Caldanaerobacter subterraneus (also known as Thermoanaerobacter tengcongensis, PDB ID 5JRX [36]). Key structural features include a small subdomain consisting of three helices (residues 1-61) and a large subdomain consisting of four helices, a four-stranded $\beta$ sheet and the heme pocket (residues 62-182, Figure 2). The structures align remarkably well considering there 
is relatively low sequence identity (less than $30 \%$ in pairwise comparisons). Major differences lie mostly in the loop connecting small-domain helices B and C, large-domain $\beta$ strands 3 and 4 and the packing between sub-domains.

\section{Structure determination of Sw H-NOX in complex with CO and IWP-051}

Previously, we showed that stimulator compounds bind near both the coiled-coil and $\alpha \mathrm{H}$ NOX domains in SGC, and that IWP-051 interacts with bacterial H-NOX proteins in a manner similar to that for heterodimeric SGC, but with much weaker binding affinity [11]. In chemical shift perturbation studies, titration of IWP-051 to the ${ }^{15} \mathrm{~N}$-labeled Sw H-NOX caused shifting of peaks in the ${ }^{1} \mathrm{H}-{ }^{15} \mathrm{~N}$ HSQC spectrum at residues E16, F17, G18, E27, L66, L69, G71, and K73, without much linewidth broadening, indicating fast exchange binding dynamics [11]. Using transferred NOESY (TrNOESY), we found IWP-051 to occupy a single conformation on binding to bacterial H-NOX proteins and sGC [11].

To better understand binding, we determined the structure of Sw H-NOX bound to IWP051 using NMR spectroscopy. Sw H-NOX saturated with IWP-051 yielded an ${ }^{1} \mathrm{H}-{ }^{15} \mathrm{~N}$ HSQC spectrum similar to that for the protein alone and was stable during data collection (Figure S1). We included $\sim 10 \%$ DMSO-d6 to overcome the relatively low solubility of IWP-051 in water. DMSO alone led to small shifts ( 0.015 ppm) throughout the ${ }^{1} \mathrm{H}-{ }^{15} \mathrm{~N}$ HSQC spectrum, while DMSO + IWP-051 led to much larger shifts [11]. In contrast, addition of phosphodiesterase inhibitor PF04447943 in DMSO, which does not stimulate sGC but has a similar chemical composition to IWP-051, does not lead to additional shifts above the DMSO background, as previously described [11]. These data indicate IWP-051 occupies a specific binding site on Sw H-NOX.

To determine the structure of Sw H-NOX bound to IWP-051, a 5-fold excess of unlabeled compound was incubated overnight with ${ }^{13} \mathrm{C},{ }^{15} \mathrm{~N}$-labeled $\mathrm{Sw} \mathrm{H}-\mathrm{NOX}$ in its $\mathrm{Fe}(\mathrm{II})-\mathrm{CO}$ state. Data were measured for ${ }^{15} \mathrm{~N}-\mathrm{HSQC},{ }^{13} \mathrm{C}-\mathrm{HSQC}, 2 \mathrm{D}-[\mathrm{F} 1, \mathrm{~F} 2]{ }^{13} \mathrm{C}^{15} \mathrm{~N}$-filtered NOESY [37], 3D ${ }^{15} \mathrm{~N}$-Edited 
NOESY and 3D ${ }^{13} \mathrm{C}$-Edited NOESY. A set of NH RDCs was measured with the ${ }^{15} \mathrm{~N}$-labeled Sw H-NOX saturated by 5-fold excess of IWP-051 for dipolar coupling restraints. The majority of peaks in the HSQC and NOESY spectra were unchanged upon binding IWP-051, indicating that the protein maintained its overall native fold. We therefore used the assigned backbone resonances of unliganded Sw H-NOX for the dihedral restraints in the initial structure calculation. Significant shifts of NOESY peaks, due to the chemical shift perturbation on the protein amides, were found in the 3D ${ }^{15} \mathrm{~N}$-Edited NOESY spectrum for the IWP-051-bound protein at residues G8, L12, E16, F17, G18, E27, L66, L69, G71, and, K73. Similar proton-proton NOESY cross peak patterns were found in these residues when compared to the unliganded protein, which allowed us to further confirm and assign the ${ }^{15} \mathrm{~N}$-Edited NOESY peaks for the IWP-051 complex. The ${ }^{13} \mathrm{C}$ Edited NOESY from the IWP-051-bound protein showed relatively small chemical shift changes. The differences in the NOESY with or without IWP-051 were detected through careful inspection of the spectrum projections along the carbon or nitrogen dimension as well as spectrum segments of each residue, and entered into the refinement restraints table.

To characterize IWP-051 and heme in the complex, a 2D-[F1,F2], ${ }^{13} \mathrm{C}^{15} \mathrm{~N}$-filtered NOESY spectrum [37] was obtained by mixing unlabeled IWP-051 with ${ }^{13} \mathrm{C},{ }^{15} \mathrm{~N}$-labeled Sw H-NOX (5:1 molar ratio, $100 \mathrm{~ms}$ mixing time). Heme is largely unlabeled in this sample due to the addition of unlabeled heme precursor (aminolevulinic acid) during expression. The spectrum showed similar NOESY cross peaks for IWP-051 (Figure S2) as found previously in TrNOESY spectrum [11], which indicated that a similar orientation/conformation is used by IWP-051 to bind to sGC and to the H-NOX proteins. The NOESY spectrum also showed similar intra-heme NOE cross peaks as was found in the unliganded protein (Figure S2), suggesting a similar heme environment in the IWP-051-bound protein.

The initial structure of the IWP-051-bound Sw H-NOX was first calculated without IWP051 by taking into account the new NOE restraints arising from binding of IWP-051, the dihedral restraints from the native protein, the new set of NH RDCs, and the re-evaluated hydrogen bond 
restraints by the AUDANA algorithm. Due to weak binding of the ligand and the overcrowded NOESY spectrum, it is difficult to obtain unambiguous assignments for the Inter-IWP-051-protein NOEs from the protein NOESY spectra. To overcome this difficulty, a 2-flurobenzyl- ${ }^{13} \mathrm{C}_{6}$-labeled IWP-051 was synthesized and a 3D ${ }^{13} \mathrm{C}$-Edited NOESY spectrum was recorded on this compound mixed with a ${ }^{15} \mathrm{~N}$-labeled $\mathrm{Sw} \mathrm{H}-\mathrm{NOX}$ in $100 \% \mathrm{D}_{2} \mathrm{O}$ buffer (5:1 molar ratio, $100 \mathrm{~ms}$ mixing time). The ${ }^{1} \mathrm{H}$ and ${ }^{13} \mathrm{C}$ resonances for the 2 -flurobenzyl- ${ }^{13} \mathrm{C}_{6}$-labeled IWP-051 can be clearly assigned using the ${ }^{1} \mathrm{H}-{ }^{13} \mathrm{C}$ HSQC,${ }^{13} \mathrm{C}$-Edited NOESY and previously recorded 2D-COSY, 2D-TOCSY, and 2D-NOESY (Figure S2) $[9,11]$. The Inter-IWP-051-protein NOEs were assigned from the 3D ${ }^{13} \mathrm{C}$ Edited NOESY (Figure S3). The initial orientation of IWP-051 was obtained by the strong NOEs from residues L12, E16, V66, and L69 to the 2-flurobenzyl ring, and the pyrazole, isoxazole and pyrimidine rings of IWP-051 were restrained by the intra-IWP-051 NOEs obtained from the 2D$[\mathrm{F} 1, \mathrm{~F} 2],{ }^{13} \mathrm{C}^{15} \mathrm{~N}$-filtered NOESY (Figure S2). The structure was refined in the same manner as for the unliganded protein, using Xplor-NIH [19]. 20 structures with the best agreement to the NMR restraints (no NOE violations $>0.5 \AA$ and no dihedral angle violations $>5^{\circ}$ ) were selected from 200 random initial structures to represent the structure ensembles (Figure 2b).

The structure ensembles showed only one major conformation for IWP-051 with the 2fluorobenzyl ring inserting into a narrow opening formed by the side chains of L12, E16 and L69 in the interface of the two sub-domains. Aside the opening is a wider hydrophobic pocket at the protein surface, formed by residues F17, V61, and L66 (Figure 3). The nitrogen atoms from pyrimidine and pyrazole ring contact the carboxylate side chains of D15 and E16, while the oxygen atom from the isoxazole ring contacts the $\varepsilon$-amino group of $\mathrm{K} 72$, which may lead to formation of hydrogen bonds or favorable electrostatic interactions at the binding site. This arrangement is consistent with the residues found to exhibit substantial chemical shift perturbations on binding IWP-051, which include E14, E16, F17, G18, L69, G71, and K73 [11].

The IWP-051 conformation found here for binding to Sw H-NOX is also consistent with that expected upon binding to sGC based on TrNOESY measurements [11]. Importantly, the 
fluorobenzyl ring, which inserts into a crevasse between subdomains in Sw H-NOX, is the position

in stimulator compounds least tolerant of change [9]. Compounds YC-1, BAY 41-2272 and Riociguat all retain this benzyl ring (Figure 1), as does proliciguat.

\section{Comparison of ligand-free and IWP-051-bound Sw H-NOX structures}

The structure ensembles for both ligand-free and IWP-051-bound Sw H-NOX display good stereochemistry, internal consistency and high agreement with RDC measurements $\left(R_{\text {dip }}=4.8 \%\right.$, correlation 0.996 , and $\mathrm{R}_{\text {dip }}=2.7 \%$, correlation 0.998 , respectively; Table S1). Overall, differences between the structures are modest, generally displaying pairwise RMSD for backbone atoms of about $1 \AA$ for the full protein, a value that drops to $\sim 0.7 \AA$ for the C-terminal (heme) subdomain. The smaller $\mathrm{N}$-terminal subdomain displays greater variability, particularly for helix $\alpha \mathrm{B}$ and the loop connecting to helix $\alpha \mathrm{C}$.

Additional analysis revealed the two subdomains in Sw H-NOX move toward one another on binding IWP-051, closing the binding pocket. Superposing the models using only the Cterminal subdomain (residues 62-182) highlights the change in subdomain interface, leading to backbone displacements in the small subdomain of up to $2.5 \AA$ (Figure 4). This closed conformation leads to an improved binding pocket by bringing residues contacting each face of IWP-051 closer together (Figure 4b). Residues F17, S59 and V61, which form the upper surface of the binding pocket, also move closer to the binding site. Closure resulted in a decrease in binding pocket surface area of $\sim 17 \AA^{2}$ and pocket volume of $\sim 9 \AA^{3}$ as assessed using the CASTp algorithm [38].

The heme-protein NOE distance restraints allowed for well-defined heme placement in both structures. On binding IWP-051, the heme orientation is unchanged, but heme position is displaced by $\sim 0.5 \AA$ toward the distal heme pocket, accompanied by a similar shift in the proximal histidine (Figure 4c). Heme displacement is likely due to shifting of helix $\alpha \mathrm{A}$ of the small 
subdomain upon IWP-051 binding. Helix $\alpha \mathrm{A}$ lies on the edge of the heme distal pocket, where it contacts the distal face of heme through N-terminal residue M1. Binding pocket closure causes helix $\alpha \mathrm{A}$ to translate along the distal pocked and away from the heme. Heme translation allows for the hydrophobic contact between the M1 side chain and the heme face to be maintained. On the other side of the distal pocket, L146 repacks to accommodate the new heme position. The L146 side chain contacts both the heme face and CO.

\section{The IWP-051 binding pocket is conserved among H-NOX proteins}

We investigated whether the binding pocket uncovered in Sw H-NOX was unique or common among H-NOX domains. Using the CASTp algorithm [38], we searched for pockets in the H-NOX structures from Nostoc sp (PDB entry 2O0G [31]), Shewanella oneidensis (PDB entry 2KII [34]), and Kordia algicida (PDB entry 6BDD [36]). Surface pockets similar to that for Sw HNOX were identified in all three $\mathrm{H}-\mathrm{NOX}$ structures, suggesting this pocket is a conserved feature of H-NOX proteins (Figure 5a). We went on to examine binding of IWP-051 to Ns H-NOX (Fe(II)CO state) using chemical shift perturbation (CSP) measurements. Titration with IWP-051 led to concentration-dependent CSPs (Figure S5), similar to those we previously observed for Sw HNOX [11]. Additionally, the CSPs from Ns H-NOX showed fast exchange binding dynamics similar to those for Sw H-NOX.

We next turned to multiple sequence alignments to search for sequence conservation in the binding pocket and extending our analysis to mammalian $\mathrm{H}-\mathrm{NOX}$ domains, which do not have high-resolution structures available. Although sequence identity among these proteins is low, sequence similarity in key regions is higher. The pockets identified by CASTp generally include the same residues, indicated by triangles in Figure 5b. Of the residues that contact IWP-051 in the Sw H-NOX complex (indicated by red triangles in Figure $5 b$ ), sequence similarity is relatively low. We conclude that while the binding pocket is a conserved feature of H-NOX proteins, pocket 
chemistry is not, which would likely lead to high variability in stimulator compound affinity among members of the H-NOX family.

\section{IWP-051 induces a change in Sw H-NOX backbone dynamics}

Protein conformational dynamics are central to protein function. For H-NOX proteins, binding of gaseous ligands such as NO leads to conformational changes that are key for signal transduction, whether it be to binding partners with the bacterial H-NOX proteins [18, 39], or to domain contacts leading to enhanced cyclase activity in SGC [14-16]. In SGC, binding of stimulator compounds is thought to induce similar changes to those induced on NO binding [5]. Backbone dynamical changes may play a role in H-NOX signal transduction but have not been previously reported.

To begin, we undertook NMR ${ }^{15} \mathrm{~N}$-relaxation and ${ }^{15} \mathrm{~N}\left[{ }^{1} \mathrm{H}\right]$ heteronuclear NOE measurements to probe the internal motion of individual residues within Sw H-NOX in its unliganded state. We examined the $\mathrm{R}_{1}{ }^{15} \mathrm{~N}$ longitudinal relaxation rates, which are sensitive to fast (picosecond to nanosecond) backbone motions [40], and found uniform backbone dynamics throughout the protein except for increased flexibility in two loop regions, spanning residues 2845 and $88-91$ (Figure $6 a$ ). The $\mathrm{R}_{2}{ }^{15} \mathrm{~N}$ transverse relaxation rates, which not only respond to fast (nanosecond) motions but also to slower (microsecond to millisecond) chemical exchange processes, were also uniform throughout the protein except for residues in loop 28-45. In this loop, $\mathrm{R}_{2}$ values nearly doubled for residues Q32, V40, and H45 (Figure 6b). Finally, we measured ${ }^{15} \mathrm{~N}\left[{ }^{1} \mathrm{H}\right]$ heteronuclear NOE, which provide information about individual backbone dynamics and is sensitive to fast (picosecond) backbone motions. Residues undergoing faster dynamics show a decrease in NOE intensity, measured as a ratio of peak intensities with and without saturation. Here again, residues 28-45 displayed increased backbone dynamics (Figure 6c). Importantly, on examining structures of $\mathrm{H}-\mathrm{NOX}$ proteins determined by X-ray crystallography, the backbone atoms for residues 28-45 are generally well-determined and do not display high disorder with the 
exception of residue 32, which in some cases is poorly ordered. Sidechains for this region often display disorder in crystal structures and the backbone conformations for this stretch vary widely, suggesting crystal contacts may stabilize one of several conformations for this region of the protein. Here, using NMR structure determination (Figure 2) and dynamics measurements (Figure 6), we observe higher variability in residues 28-45.

Binding of IWP-051 did not greatly alter Sw H-NOX dynamics. A similar $\mathrm{R}_{1}$ distribution was observed with the exception that the loop region 88-91 gained more flexibility (Figure 6a), a region well-away from the binding site. In R2 measurements, loop 28-45 displayed somewhat dampened dynamics, particularly for residues Q32, E34 and H45 (Figure 6b). HetNOE values (Figure 6c) decreased at the binding site, including those for G18, S59, V61, and L66, indicating IWP-051 binding leads to greater backbone motion at the binding site. Interestingly, similar residues, including E16, F17, and E20, are involved in Sw H-NOX recognition of its binding partner, cyclic-di-GMP synthase [18]. Flexibility in this region may facilitate binding.

\section{Comparison with photoaffinity labeling and cryo-EM studies}

Although high resolution structural data for stimulator binding to sGC are still lacking, insight into binding location come from photoaffinity covalent labeling combined with mass spectrometry [11], and from single-particle cryo-electron microscopy [15]. Photolabeling was conducted with a stimulator compound modified to have a diazirine moiety attached to the pyrimidine ring, where modifications are well tolerated, and photoactivation leads to covalent attachment to nearby residues in the protein. For sGC from Manduca sexta, these data indicate that stimulators bind near both the $\beta \mathrm{H}-\mathrm{NOX}$ domain (residues 6-9) and the coiled-coil domain ( $\beta$ chain residues 361-362 and 365-366). A recent cryo-EM study of SGC bound to YC-1, also using the protein from Manduca sexta, yielded a backbone model of sGC at $5.8 \AA$ resolution with density 
attributed to YC-1 in two possible orientations. In this model, YC-1 was within $5 \AA$ of $\beta \mathrm{H}-\mathrm{NOX}$ residues V39, F77, C78, and Y112, as well as coiled-coil residues $\beta$ Q349 and $\alpha$ 422-427.

Our position for IWP-051 in Sw H-NOX is consistent with both models, although not perfectly overlapping. In particular, four of five of the residues either labeled through photoaffinity or identified by cryo-EM in the H-NOX domain, lie along the same subdomain interface identified for binding in the NMR structure, and the fifth residue is nearby. However, the binding site found for $\mathrm{Sw} \mathrm{H}-\mathrm{NOX}$ is translated a few angstroms along the interface, moving away from the heme distal pocket, with respect to the cryo-EM and photoaffinity labeling studies.

\section{Discussion}

We have determined the NMR structure of Sw H-NOX in the presence and absence of an sGC stimulator, IWP-051, to high precision. Spectra were measured with heme in the ferrous (Fe II) state and bound to $\mathrm{CO}$, yielding a diamagnetic system amenable to full structure determination. The protein displays the expected overall H-NOX fold, with a small N-terminal helical domain and a lager C-terminal domain of mixed $\alpha$ helix and $\beta$ sheet and a large heme pocket. The heme conformation is modestly distorted from planar and is linked to the protein through a proximal histidine, $\mathrm{H} 104$. The distal pocket is hydrophobic and with extra room, typical of H-NOX proteins. The sidechain for L146, however, is in contact with both CO and heme.

Stimulator IWP-051 binds only weakly to Sw H-NOX $\left(K_{d} \sim 1 \mathrm{mM}\right)$, unlike for sGC where binding is tighter and stimulates $\mathrm{SGC}$ in $\mathrm{HEK}$ cells with an $\mathrm{EC}_{50}$ value of $290 \mathrm{nM}$ [9]. Binding to Sw H-NOX is, however, specific and involves the same compound conformation as for binding to sGC [11], with the benzyl and pyrimidine rings perpendicular to the core pyrazole ring, and the isoxazole ring rotated slightly out-of-plane with respect to the pyrazole ring. The benzyl ring, which is the position least tolerant to change in SGC stimulators (Figure 1; [7]), inserts between the large and small subdomains, contacting residues L12, E16 and L69. The pyrazole ring is also 
near E16 and in an arrangement that could lead to hydrogen bond formation if E16 were protonated. The isoxazole and pyrimidine rings are less in contact with the protein, but are close to $\mathrm{K} 72$ and $\mathrm{D} 15$, respectively. Overall, binding interactions are modest, consistent with the weak affinity of IWP-051 for Sw H-NOX. Binding does however induce closure of the binding pocket through an $\sim 2.5 \AA$ shift in small-subdomain position with respect to large subdomain. This rotation is similar to that observed in the H-NOX from Shewanella oneidensis on binding NO $[1,35]$.

In addition to subdomain movements, dynamical properties of $\mathrm{H}-\mathrm{NOX}$ backbone residues are likely of importance in forming productive complexes with binding partners, and in signal transduction in multidomain proteins such as sGC. Here, we show that Sw H-NOX has relatively uniform backbone dynamics across both subdomains with the exception of the large loop between helices $\alpha \mathrm{B}$ and $\alpha \mathrm{C}$ (residues 28-45, small subdomain) and the loop between helices $\alpha \mathrm{E}$ and $\alpha \mathrm{F}$ (residues 88-91, large subdomain), both of which show enhanced dynamics compared to the overall protein. Dynamics in these regions increase on binding IWP-051, as do dynamics in the binding site. Interestingly, residues 38 and 40 directly contact the sGC coiled coil in the activated conformation observed by cryo-EM, but not in the inactive form $[14,15]$.

How stimulator binding to Sw H-NOX relates to binding in full-length SGC remains unknown, but certain aspects are becoming clear. Importantly, both photochemical labeling [11] and cryo-EM [15] support a model in which stimulator compounds bind at the interface of the $\mathrm{H}$ NOX and coiled-coil domains, and therefore stand-alone H-NOX domains contain only a portion of the binding site. Nonetheless, the binding pocket identified in Sw H-NOX appears to be conserved among H-NOX proteins (Figure 5). Binding to the subdomain interface, as we report here for Sw H-NOX, appears to also occur in SGC, although possibly to a position somewhat shifted toward the heme distal pocket. Additionally, movement at the subdomain interface as occurs for Sw H-NOX upon binding IWP-051 is also seen on activation of SGC when examined by cryo-EM [14]. Interestingly, mutations introduced into the subdomain interface can either block 
stimulator binding, as seen with the L12W/T48W double mutant in Manduca sGC [11], or mimic stimulator action, as seen in the I52W/L67W double mutant, also in Manduca sGC*.

\section{Materials and methods}

Detailed methods are described in Supporting Information.

\section{Protein expression, purification, and NMR sample preparation}

Proteins were expressed in E. coli and purified using a His-tag fused to the protein followed by cleavage of the tag with TEV protease, as previously described [11]. Isotope enrichment was accomplished using M9 media isotopically enriched for ${ }^{15} \mathrm{~N}$ or ${ }^{13} \mathrm{C} /{ }^{15} \mathrm{~N}$. Full protocols for protein isolation and preparation for NMR experiments are described in the Supplemental information text.

\section{NMR spectroscopy}

NMR experiments were performed at $293 \mathrm{~K}$ on either Agilent $800-\mathrm{MHz}$ or Bruker $600,800-$ $\mathrm{MHz}$ Avance NEO spectrometers, all equipped with triple resonance cryogenic probes. Samples for NMR measurements typically contained 0.5-0.8 mM protein in buffer containing $90 \% \mathrm{H}_{2} \mathrm{O} / 10 \%$ $\mathrm{D}_{2} \mathrm{O}$ or $100 \% \mathrm{D}_{2} \mathrm{O}$, and $50 \mathrm{mM} \mathrm{Na} / \mathrm{KPO}_{4}, 50 \mathrm{mM} \mathrm{NaCl}$ at $\mathrm{pH}$ 7.4. The $\mathrm{Fe}(\mathrm{II})-\mathrm{CO}$ complex was prepared by reducing heme with dithionite followed by saturation with $\mathrm{CO}$, as previously described [11] and in the Supplemental Information text. IWP-051 was dissolved in 100\% DMSO-d6 and 25-50 mM was prepared as a stock solution. For the Sw H-NOX IWP-051 complex, a 5-fold excess amount of IWP-051 was used in the NMR measurements, which resulted in $\sim 10-15 \%$ DMSO-d6. Both labeled and unlabeled IWP-051 were generously provided by Ironwood Pharmaceuticals.

NOE distance restraints, dihedral angle, hydrogen bond, and RDC restraints 
Intramolecular NOE distance restraints for protein were obtained from $3 \mathrm{D}{ }^{15} \mathrm{~N}-\mathrm{NOESY}-$ HSQC, 3D ${ }^{13} \mathrm{C}-\mathrm{HSQC}-\mathrm{NOESY}$ (in $100 \% \mathrm{D}_{2} \mathrm{O}$ buffer) and $3 \mathrm{D}{ }^{13} \mathrm{C}-\mathrm{HSQC}-\mathrm{NOESY}$ (aromatic). TALOS-N was used to predict backbone dihedral angles from chemical shifts [21]. Hydrogen bond restraints were generated using the AUDANA algorithm, which predicts hydrogen bonds from NOE cross peak patterns of secondary structures and reevaluates them after each cycle of structure calculation [22]. RDC data were obtained using $2 \mathrm{D}{ }^{1} \mathrm{H}-{ }^{15} \mathrm{~N}$ correlation via TROSY allowing for TROSY and AntiTROSY signal in an IPAP manner [41]. The RDC constants were obtain by the differences between TROSY and AntiTROSY components from unaligned and pf1 phage-aligned samples.

\section{Structure determination}

NOESY peaks were assigned manually and automatically in iterative cycles of PONDEROSA-C/S's AUDANA and CYANA calculations [42, 43]. Peak intensities were calibrated and converted to distance restraints using NMRFAM-SPARKY [20]. Structure determination was accomplished using Xplor-NIH [19]. The PDB, topology and parameter files for heme and IWP051 were prepared using Open Babel [44] and RUNER [45], and using MOPAC PM7 force fields with General Amber Force Field (GAFF) options [32]. The MOPAC PM7 force field was designed using experimental and ab initio reference data for biological systems. Open Babel is a tool for format conversion. RUNER was the front end developed by the National Magnetic Resonance Facility at Madison (NMRFAM) to unify the nomenclatures using the ALATIS tool for labeling consistency [46]. PSF files were generated by Xplor-NIH. The detailed structure calculation is described in the Supplemental Information text.

\section{Chemical shift perturbation for Ns H-NOX}

CSPs were measured by collecting a series of ${ }^{1} \mathrm{H}-{ }^{15} \mathrm{~N} H S Q \mathrm{C}$ spectrum using $450 \mu \mathrm{M}{ }^{15} \mathrm{~N}$ labeled Ns H-NOX with increasing concentration of IWP-051 (100-4000 $\mu \mathrm{M})$ or DMSO-d6 (1.6- 
9.6\%). Peaks with significant chemical shift change were identified by overlaying the spectra.

The shifts due to the effects from DMSO-d6 were ignored.

\section{${ }^{15} \mathrm{~N}$ relaxation and ${ }^{15} \mathrm{~N}\left[{ }^{1} \mathrm{H}\right]$-heteronuclear NOE (hetNOE) measurements}

The ${ }^{15} \mathrm{~N}$ longitudinal $\left(\mathrm{R}_{1}\right)$ and transverse $\left(\mathrm{R}_{2}\right)$ relaxation rates, and ${ }^{15} \mathrm{~N}\left[{ }^{1} \mathrm{H}\right]$ heteronuclear

NOE, were measured in an interleaved manner as in the TROSY-based experiments [47] at 800

$\mathrm{MHz}$. A recycle delay of $3.0 \mathrm{~s}$ between experiments was used along with the following relaxation delays for T1: 50, 200, 400, 800, 1200, 1600, 2000, 2500, $3000 \mathrm{~ms}$; and for T2: 8.5, 17.0, 25.5, $33.9,42.4,50.9,67.87,84.84,101.81$, and $118.8 \mathrm{~ms}$. The spectra were analyzed and plotted by NMRFAM-Sparky to obtain the rates for each residue. The ${ }^{15} \mathrm{~N}\left[{ }^{1} \mathrm{H}\right]-\mathrm{NOE}$ was determined from a pair of interleaved spectra acquired with or without proton presaturation at $800 \mathrm{MHz}$. Values of hetNOE were obtained by ratio of the peak intensities for each residue.

\section{Accession numbers}

Coordinates for the Sw H-NOX structures are deposited in the Protein Data Bank under PDB entries 6OCV and 6WQE. NMR assignment data are deposited in the Biological Magnetic Resonance Data Bank under BMRB entry 27284.

\section{Supplemental data}

Supplementary text and data to this article can be found online at: XXX.

\section{Acknowledgments}

We are grateful to Michael Clarkson for help with NMR measurements at the University of Arizona, and to Ironwood Pharmaceuticals for providing labeled and unlabeled IWP-051. This study was supported by National Institutes of Health Grants R01 GM117357, P30 CA023074 and U54 CA143924 (to W. R. M.) and T32 HL007249 (to C. C.). This study was also supported by 
Grants 17POST33670593 (to C. C.) from the American Heart Association and by Sponsored Research Agreement 100003104 from Ironwood Pharmaceuticals (to W. R. M.). This study made use of the National Magnetic Resonance Facility at Madison, which is supported by NIH grants P41 GM103399 (NIGMS) and P41 GM66326 (NIGMS). Additional equipment was purchased with funds from the University of Wisconsin, the NIH (RR02781, RR08438), the NSF (DMB8415048, OIA-9977486, BIR-9214394), and the USDA.

\section{Footnotes}

Double mutation (I52W/L67W) in the heme domain of soluble guanylyl cyclase mimics stimulator compounds, Jessica A. Kievenaar, Andrzej Weichsel, Jacob T. Croft, Jinghui Li, Changjian Feng, and William R. Montfort, manuscript in preparation.

\section{Conflict of Interest}

The authors declare no conflicts of interest.

\section{Author Contributions}

Cheng-Yu Chen: Conceptualization, Investigation, Formal analysis, Methodology, Funding acquisition, Writing- Original draft preparation, Reviewing and Editing. Woonghee Lee: Investigation, Formal analysis, Methodology, Writing- Reviewing and Editing. William R. Montfort: Conceptualization, Project administration, Funding acquisition, Formal analysis, Writing- Original draft preparation, Reviewing and Editing. 


\section{Figure Legends}

Figure 1. Chemical structures of sGC stimulators. The positions of ${ }^{13} \mathrm{C}$ enrichment in IWP-051 is indicated with stars, and overall numbering is indicated. The 5-membered pyrazole ring is shown in the center (atoms 7, 14,15, 22 and 24) and isoxazole ring in the upper left of the figure (atoms 5, 6, 13, 23, 26).

Figure 2. Backbone ensemble structures of Sw H-NOX. (a) Ligand-free protein. (b) Complex with IWP-051. Secondary structure elements are labeled and heme and IWP-051 indicated with stick representation. The structures are shown in stereo (wall-eyed view). All structure figures were prepared with PyMOL (Schrodinger, LLC).

Figure 3. Binding interface between Sw H-NOX and IWP-051. (a) Surface and cartoon representation for the binding interface. Residues in the binding pocket are shown in blue. IWP051 is colored orange and shown in stick representation. (b) Close-up view of IWP-051 binding interactions, stick representation, with IWP-051 shown in orange and binding pocket residues shown in blue. The single structure closest to the average of the ensemble structures is presented.

Figure 4. Structure comparison between unliganded and IWP-051-bound Sw H-NOX. (a) Unliganded and IWP-051-bound ensemble structures after superposing using only the C-terminal heme subdomain (residues 63-182). Unliganded Sw H-NOX is colored orange and the IWP-051bound structure is colored purple. Shifting of the N-terminal subdomain on binding IWP-051 is indicated by a dashed arrow. (b) Close-up view at the IWP-051 binding site from the aligned structures (ensemble view upper left; ribbon cartoon of structures near the average structures, with labels, lower right). (c) Close-up view of the heme pocket (ensemble view upper left; ribbon cartoon, lower right). 
Figure 5. The IWP-051 binding pocket is conserved in H-NOX proteins. (a) Surface representation of the binding pockets from $\mathrm{Ns} \mathrm{H}-\mathrm{NOX}$, So $\mathrm{H}-\mathrm{NOX}$ and $\mathrm{Ka} \mathrm{H-NOX.} \mathrm{The} \mathrm{relative}$ position of IWP-051 is shown by superposing the distal subdomain (residue 1-62) of Sw H-NOX to each structure. Residues involved in the surface pocket are shown in blue stick representation and IWP-051 in orange stick representation. (b) Multiple sequence alignment of H-NOX domains, showing binding site residues. So: Shewanella oneidensis, Ns: Nostoc sp, Cs: Caldanaerobacter subterraneus, Hs sGC: human sGC $\beta$ H-NOX, Ka: Kordia algicida, Cb: Clostridium botulinum, Ms sGC: Manduca sexta $\beta$ H-NOX, Sw: Shewanella woodyi. Triangles indicate the residues forming the surface pockets calculated by CASTp, with red for Sw H-NOX, blue for Ns H-NOX, green for So H-HNOX, and purple for Ka H-NOX. Secondary structure is labeled on top with $\alpha$ helices shown as cylinders. The alignment was performed with the program T-Coffee [48]. The graphical presentation was done by the program Jalview [49] using the Clustal X color scheme [50].

Figure 6. ${ }^{15} \mathrm{~N}$ relaxation dynamics for native and IWP-051-bound Sw H-NOX. (a) ${ }^{15} \mathrm{~N}$ longitudinal relaxation rates $\left(R_{1}\right)$ plotted against residue number. (b) ${ }^{15} \mathrm{~N}$ transverse relaxation rates $\left(R_{2}\right)$ plotted against residue number. (c) ${ }^{15} \mathrm{~N}\left[{ }^{1} \mathrm{H}\right]$ heteronuclear $\mathrm{NOE}$ intensities plotted against residue number. Secondary structure elements are indicated by cylinders for $\alpha$ helices and arrows for $\beta$ strands. Data points for unliganded and IWP-051-bound proteins are shown as blue and orange dots, respectively. Error bars are the standard deviation from data fitting ( $R_{1}$ and $R_{2}$ plots). Flexible loops are highlighted with purple boxes and the IWP-051 binding site is highlighted with blue boxes. 


\section{REFERENCES}

[1] Guo Y, Marletta MA. Structural Insight into H-NOX Gas Sensing and Cognate Signaling Protein Regulation. Chembiochem. 2019, 20:7-19.

[2] Williams DE, Boon EM. Towards Understanding the Molecular Basis of Nitric Oxide-Regulated Group Behaviors in Pathogenic Bacteria. J. Innate Immun. 2019, 11:205-215.

[3] Persson A, Gross E, Laurent P, Busch KE, Bretes H, de Bono M. Natural variation in a neural globin tunes oxygen sensing in wild Caenorhabditis elegans. Nature. 2009, 458:1030-1033.

[4] Zimmer M, Gray JM, Pokala N, Chang AJ, Karow DS, Marletta MA, et al. Neurons detect increases and decreases in oxygen levels using distinct guanylate cyclases. Neuron. 2009, 61:865-879.

[5] Montfort WR, Wales JA, Weichsel A. Structure and Activation of Soluble Guanylyl Cyclase, the Nitric Oxide Sensor. Antioxid. Redox Signal. 2017, 26:107-121.

[6] Derbyshire ER, Marletta MA. Structure and regulation of soluble guanylate cyclase. Annu. Rev. Biochem. 2012, 81:533-559.

[7] Sandner P, Zimmer DP, Milne GT, Follmann M, Hobbs A, Stasch JP. Soluble Guanylate Cyclase Stimulators and Activators. Handbook of experimental pharmacology. 2019.

[8] Ko FN, Wu CC, Kuo SC, Lee FY, Teng CM. YC-1, a novel activator of platelet guanylate cyclase. Blood. 1994, 84:4226-4233.

[9] Nakai T, Perl NR, Barden TC, Carvalho A, Fretzen A, Germano P, et al. Discovery of IWP051, a Novel Orally Bioavailable sGC Stimulator with Once-Daily Dosing Potential in Humans. ACS Med. Chem. Lett. 2016, 7:465-469.

[10] Yoo BK, Lamarre I, Rappaport F, Nioche P, Raman CS, Martin JL, et al. Picosecond to second dynamics reveals a structural transition in Clostridium botulinum NO-sensor triggered by the activator BAY-41-2272. ACS Chem. Biol. 2012, 7:2046-2054. 
[11] Wales JA, Chen CY, Breci L, Weichsel A, Bernier SG, Sheppeck JE, 2nd, et al. Discovery of stimulator binding to a conserved pocket in the heme domain of soluble guanylyl cyclase. J. Biol. Chem. 2018, 293:1850-1864.

[12] Fritz BG, Roberts SA, Ahmed A, Breci L, Li W, Weichsel A, et al. Molecular Model of a Soluble Guanylyl Cyclase Fragment Determined by Small-Angle X-ray Scattering and Chemical Cross-Linking. Biochemistry. 2013, 52:1568-1582.

[13] Campbell MG, Underbakke ES, Potter CS, Carragher B, Marletta MA. Single-particle EM reveals the higher-order domain architecture of soluble guanylate cyclase. Proc. Natl. Acad. Sci. USA. 2014, 111:2960-2965.

[14] Kang Y, Liu R, Wu JX, Chen L. Structural insights into the mechanism of human soluble guanylate cyclase. Nature. 2019, 574:206-210.

[15] Horst BG, Yokom AL, Rosenberg DJ, Morris KL, Hammel M, Hurley JH, et al. Allosteric activation of the nitric oxide receptor soluble guanylate cyclase mapped by cryo-electron microscopy. eLife. 2019, 8.

[16] Weichsel A, Kievenaar JA, Curry R, Croft JT, Montfort WR. Instability in a coiled-coil signaling helix is conserved for signal transduction in soluble guanylyl cyclase. Protein Sci. 2019, 28:1830-1839.

[17] Lukin JA, Ho C. Nuclear magnetic resonance of hemoglobins. Methods Mol. Med. 2003, $82: 251-269$.

[18] Lahiri T, Luan B, Raleigh DP, Boon EM. A structural basis for the regulation of an H-NOXassociated cyclic-di-GMP synthase/phosphodiesterase enzyme by nitric oxide-bound $\mathrm{H}$ NOX. Biochemistry. 2014, 53:2126-2135.

[19] Schwieters CD, Kuszewski JJ, Clore GM. Using Xplor-NIH for NMR molecular structure determination. Prog. Nucl. Magn. Reson. Spectrosc. 2006, 48:47-62.

[20] Lee W, Tonelli M, Markley JL. NMRFAM-SPARKY: enhanced software for biomolecular NMR spectroscopy. Bioinformatics (Oxford, England). 2015, 31:1325-1327. 
[21] Shen Y, Bax A. Protein structural information derived from NMR chemical shift with the neural network program TALOS-N. Methods Mol. Biol. 2015, 1260:17-32.

[22] Lee W, Petit CM, Cornilescu G, Stark JL, Markley JL. The AUDANA algorithm for automated protein 3D structure determination from NMR NOE data. J. Biomol. NMR. 2016, 65:51-57.

[23] Powers L, Sessler JL, Woolery GL, Chance B. CO bond angle changes in photolysis of carboxymyoglobin. Biochemistry. 1984, 23:5519-5523.

[24] Vojtechovsky J, Chu K, Berendzen J, Sweet RM, Schlichting I. Crystal structures of myoglobin-ligand complexes at near-atomic resolution. Biophys. J. 1999, 77:2153-2174.

[25] Maes EM, Roberts SA, Weichsel A, Montfort WR. Ultrahigh Resolution Structures of Nitrophorin 4: Heme Distortion in Ferrous CO and NO Complexes. Biochemistry. 2005, 44:12690-12699.

[26] La Mar GN, Budd DL, Viscio DB, Smith KM, Langry KC. Proton nuclear magnetic resonance characterization of heme disorder in hemoproteins. Proc. Natl. Acad. Sci. USA. 1978, 75:5755-5759.

[27] Shokhireva T, Weichsel A, Smith KM, Berry RE, Shokhirev NV, Balfour CA, et al. Assignment of the ferriheme resonances of the low-spin complexes of nitrophorins 1 and 4 by (1)H and (13)C NMR spectroscopy: comparison to structural data obtained from X-ray crystallography. Inorganic chemistry. 2007, 46:2041-2056.

[28] Weichsel A, Andersen JF, Roberts SA, Montfort WR. Reversible nitric oxide binding to nitrophorin 4 from Rhodnius prolixus involves complete distal pocket burial. Nat. Struct. Biol. 2000, 7:551-554.

[29] Pellicena P, Karow DS, Boon EM, Marletta MA, Kuriyan J. Crystal structure of an oxygenbinding heme domain related to soluble guanylate cyclases. Proc. Natl. Acad. Sci. USA. 2004, 101:12854-12859.

[30] Nioche P, Berka V, Vipond J, Minton N, Tsai AL, Raman CS. Femtomolar sensitivity of a NO sensor from Clostridium botulinum. Science. 2004, 306:1550-1553. 
[31] Ma X, Sayed N, Beuve A, van den Akker F. NO and CO differentially activate soluble guanylyl cyclase via a heme pivot-bend mechanism. EMBO J. 2007, 26:578-588.

[32] Stewart JJP. Optimization of parameters for semiempirical methods VI: more modifications to the NDDO approximations and re-optimization of parameters. J. Mol. Model. 2013, 19:132.

[33] Tian Y, Schwieters CD, Opella SJ, Marassi FM. High quality NMR structures: a new force field with implicit water and membrane solvation for Xplor-NIH. J. Biomol. NMR. 2017, 67:3549.

[34] Erbil WK, Price MS, Wemmer DE, Marletta MA. A structural basis for H-NOX signaling in Shewanella oneidensis by trapping a histidine kinase inhibitory conformation. Proc. Natl. Acad. Sci. USA. 2009, 106:19753-19760.

[35] Herzik MA, Jr., Jonnalagadda R, Kuriyan J, Marletta MA. Structural insights into the role of iron-histidine bond cleavage in nitric oxide-induced activation of $\mathrm{H}-\mathrm{NOX}$ gas sensor proteins. Proc. Natl. Acad. Sci. USA. 2014, 111:E4156-E4164.

[36] Hespen CW, Bruegger JJ, Phillips-Piro CM, Marletta MA. Structural and Functional Evidence Indicates Selective Oxygen Signaling in Caldanaerobacter subterraneus H-NOX. ACS Chem. Biol. 2016, 11:2337-2346.

[37] Ogura K, Terasawa H, Inagaki F. An improved double-tuned and isotope-filtered pulse scheme based on a pulsed field gradient and a wide-band inversion shaped pulse. J. Biomol. NMR. 1996, 8:492-498.

[38] Tian W, Chen C, Lei X, Zhao J, Liang J. CASTp 3.0: computed atlas of surface topography of proteins. Nucleic Acids Res. 2018, 46:W363-W367.

[39] Plate L, Marletta MA. Nitric oxide-sensing H-NOX proteins govern bacterial communal behavior. Trends Biochem. Sci. 2013, 38:566-575.

[40] Kleckner IR, Foster MP. An introduction to NMR-based approaches for measuring protein dynamics. Biochim. Biophys. Acta. 2011, 1814:942-968. 
[41] Nietlispach D. Suppression of anti-TROSY lines in a sensitivity enhanced gradient selection TROSY scheme. J. Biomol. NMR. 2005, 31:161-166.

[42] Lee W, Stark JL, Markley JL. PONDEROSA-C/S: client-server based software package for automated protein 3D structure determination. J. Biomol. NMR. 2014, 60:73-75.

[43] Guntert P, Buchner L. Combined automated NOE assignment and structure calculation with CYANA. J. Biomol. NMR. 2015, 62:453-471.

[44] O'Boyle NM, Banck M, James CA, Morley C, Vandermeersch T, Hutchison GR. Open Babel: An open chemical toolbox. J. Cheminform. 2011, 3:33.

[45] Dashti H, Wedell JR, Corniliescu G, Schwieters CD, Westler WM, Markley JL, et al. Robust nomenclature and software for enhanced reproducibility in molecular modeling of small molecules. Biorxiv. 2018:doi: https://doi.org/10.1101/429530.

[46] Dashti H, Westler WM, Markley JL, Eghbalnia HR. Unique identifiers for small molecules enable rigorous labeling of their atoms. Sci. Data. 2017, 4:170073.

[47] Zhu G, Xia Y, Nicholson LK, Sze KH. Protein dynamics measurements by TROSY-based NMR experiments. J. Magn. Reson. 2000, 143:423-426.

[48] Notredame C, Higgins DG, Heringa J. T-Coffee: A novel method for fast and accurate multiple sequence alignment. J. Mol. Biol. 2000, 302:205-217.

[49] Waterhouse AM, Procter JB, Martin DM, Clamp M, Barton GJ. Jalview Version 2--a multiple sequence alignment editor and analysis workbench. Bioinformatics (Oxford, England). 2009, 25:1189-1191.

[50] Thompson JD, Gibson TJ, Plewniak F, Jeanmougin F, Higgins DG. The CLUSTAL_X windows interface: flexible strategies for multiple sequence alignment aided by quality analysis tools. Nucleic Acids Res. 1997, 25:4876-4882. 


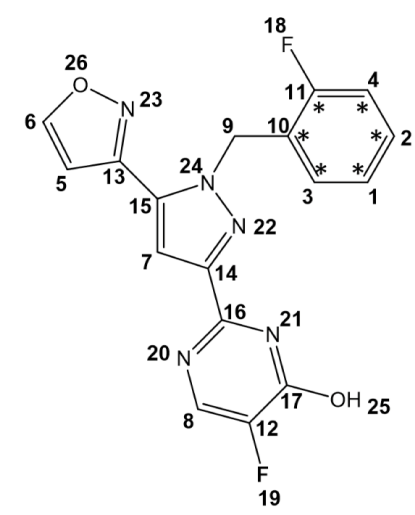

IWP-051

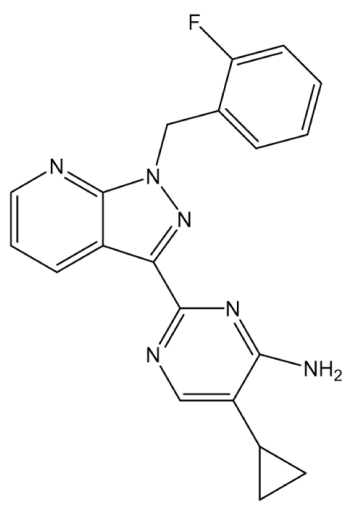

BAY 41-2272

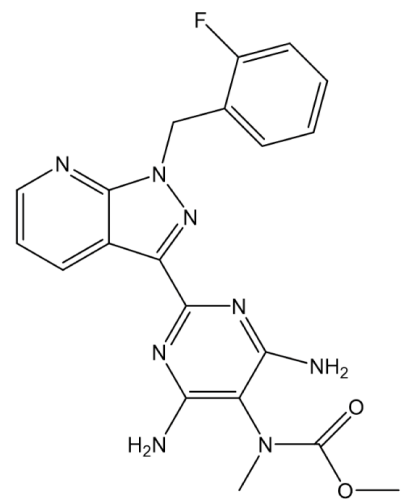

Riociguat<smiles>OCc1ccc(-c2nn(Cc3ccccc3)c3ccccc23)o1</smiles>

YC-1

Figure 1 

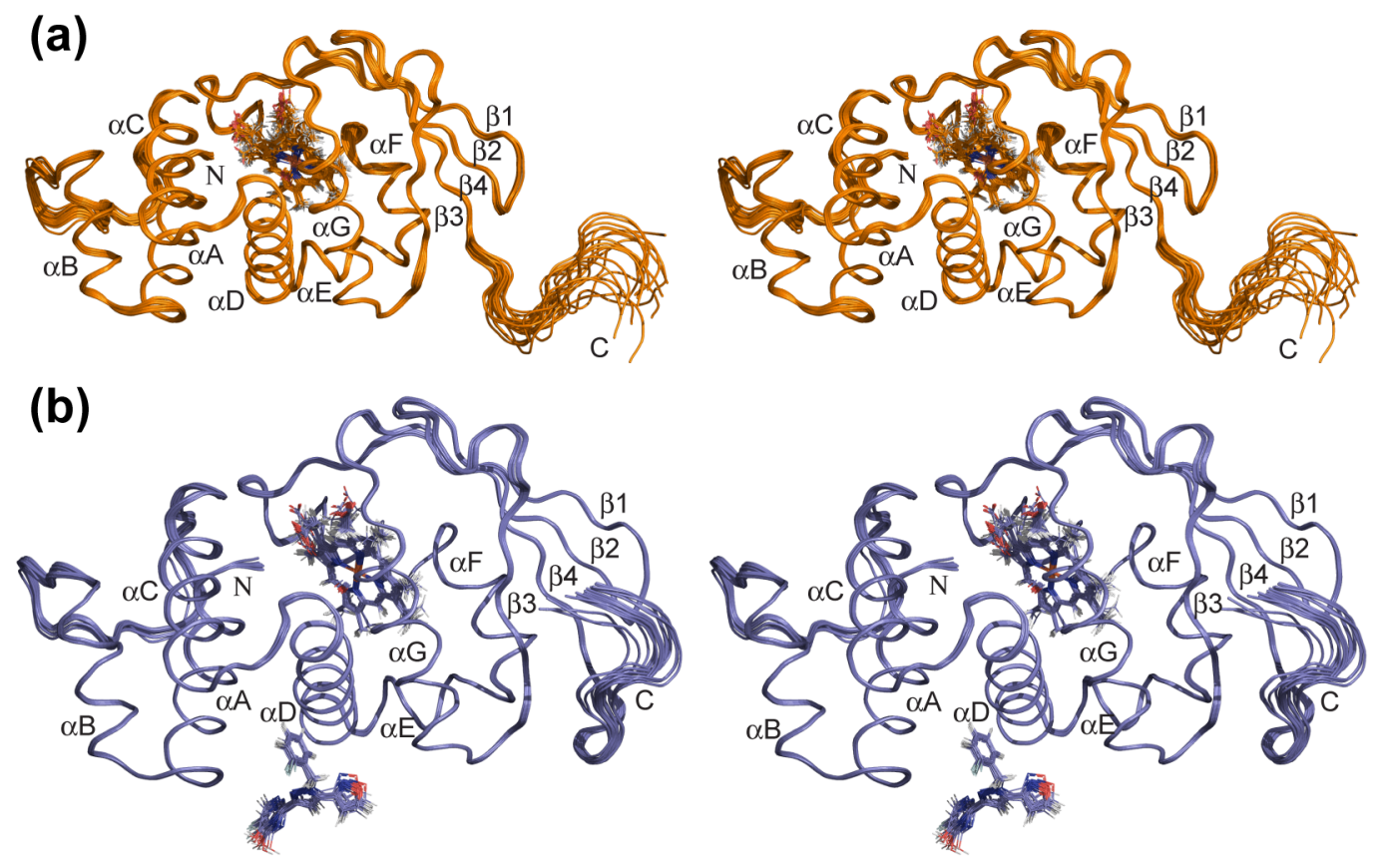

Figure 2 
(a)

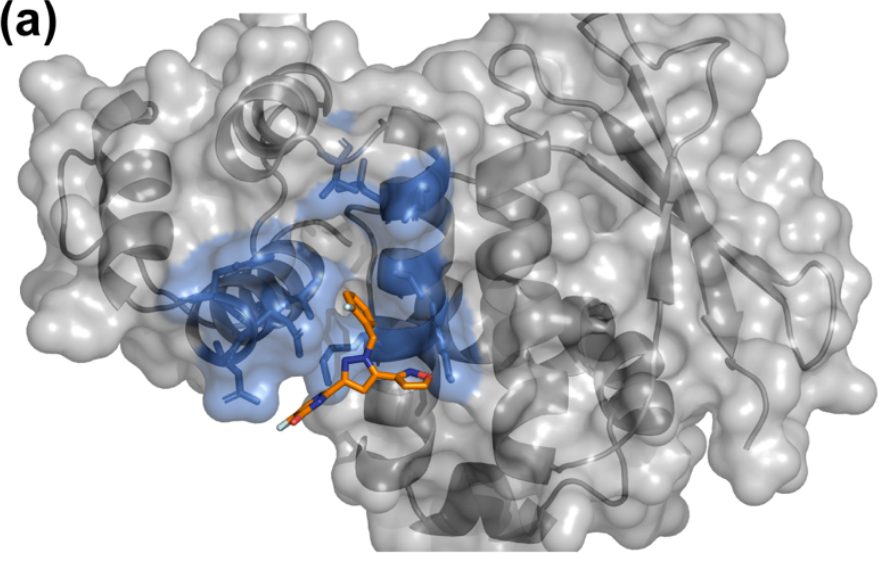

(b)

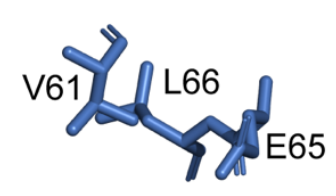

F17

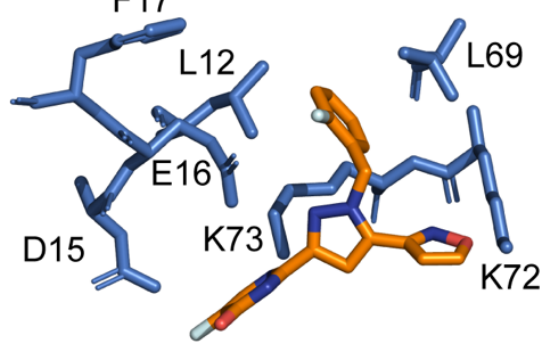

Figure 3 

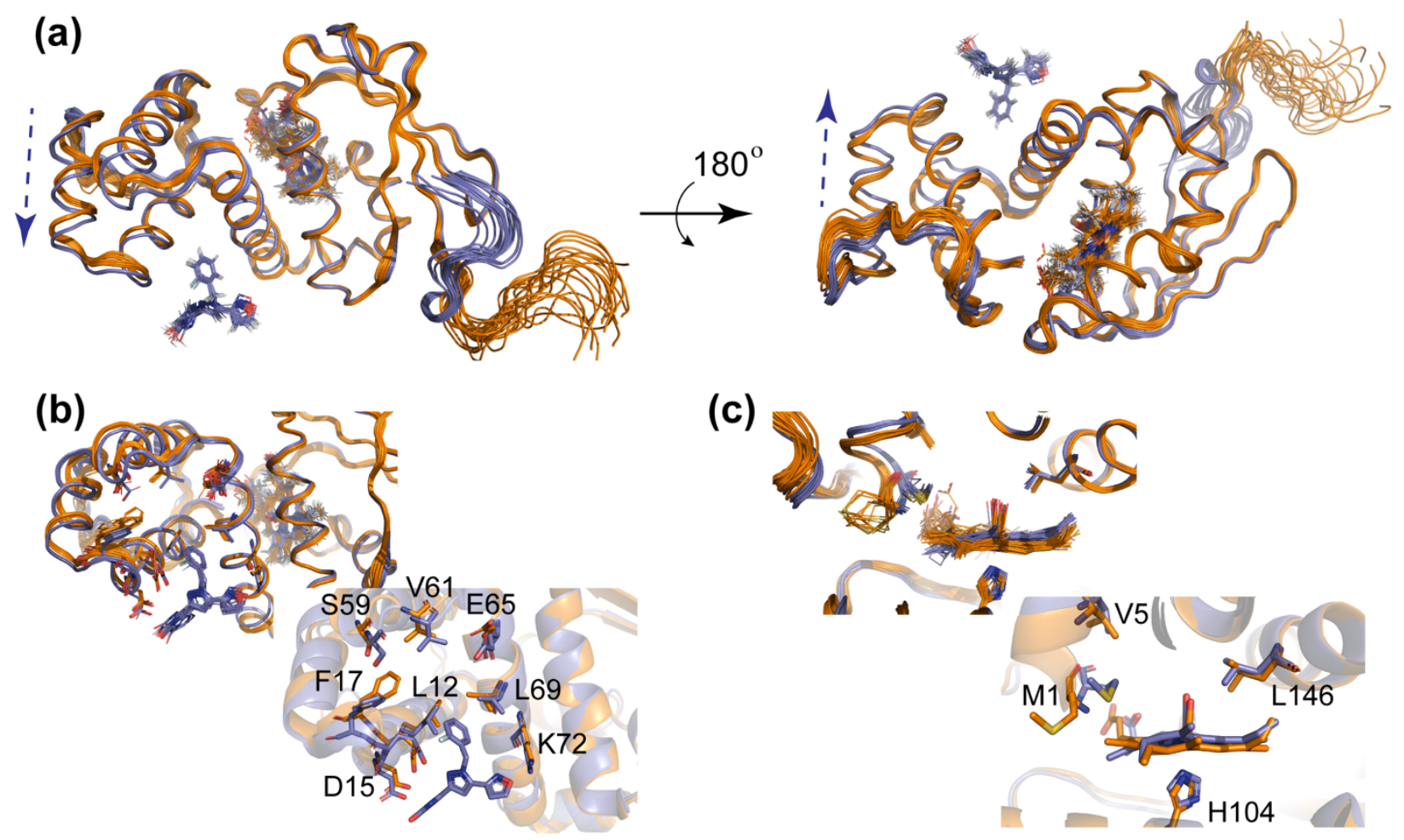

(c)

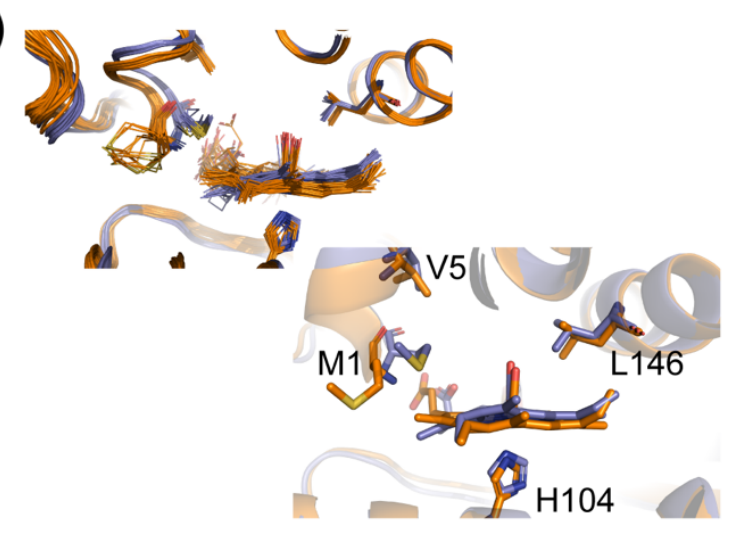

Figure 4 
(a)

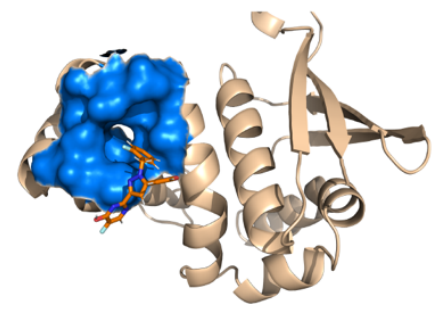

Ns H-NOX

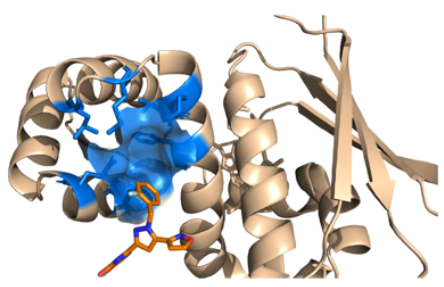

So H-NOX $\triangle$

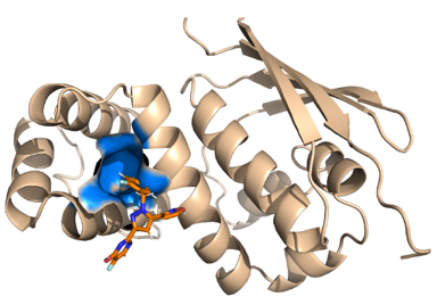

Ka H-NOX $\triangle$

(b)

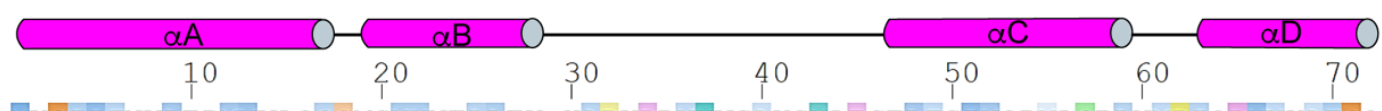

So 1 MKGIIFNVLEDMVVAQCGMSVWNELLEK-HAPKDRVYVSAKSYAESELFSIVQDVAQRLNMPIQDVVKAFGQ- 71

Ns 1 MYGLVNKAIQDMISKHHGEDTWEAIKQKAGLEDIDFFVGMEAYSDDVTYHLVGAASEVLGKPAEELLIAFGE- 77.

Cs 1 MKGTIVGTWIKTLRDLYGNDVVDESLKSVGWEPDRVITPLEDIDDDEVRRIFAKVSEKTGKNVNEIWREVGR- 72

Hs sGC 1 MYGFVNHALELLVIRNYGPEVWEDIKKEAQLDEEGQFLVRIIYDDSKTYDLVAAASKVLNLNAGEILQMFGK- 72

Ka 1 MKGIIFTEFLDLVEDKFGLEMVDKIITQSELESEGVYTSIGTYRFSEMLQLLQNLSANTDVS IDDLLLTYAE- 77.

Cb 1 MKGTVVATWVKTCRKLYGEEAVDKAMNAVGWQGK-IFSPMENVEDTKVKNMI---NEIASNVNKDVKTLWGQI 69

Ms sGC 1 MYGFVNYALELLVMKTFDEETWETIKKKADVAMEGSFLVRQIYEDEITYNLITAAVEVLQIPADAILELFGK- 72

SW 1 MMGMVETGLMELIEDEFGYETLDTLLESCELQSEGIYTSVGSYDHQELLQLVVKLSEVSSVPVTELVRLFGK- 72

${ }_{\Delta}^{\Delta \Delta} \Delta \frac{\Delta}{\Delta} \Delta$

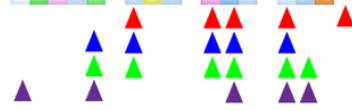

\section{Figure 5}


(a)

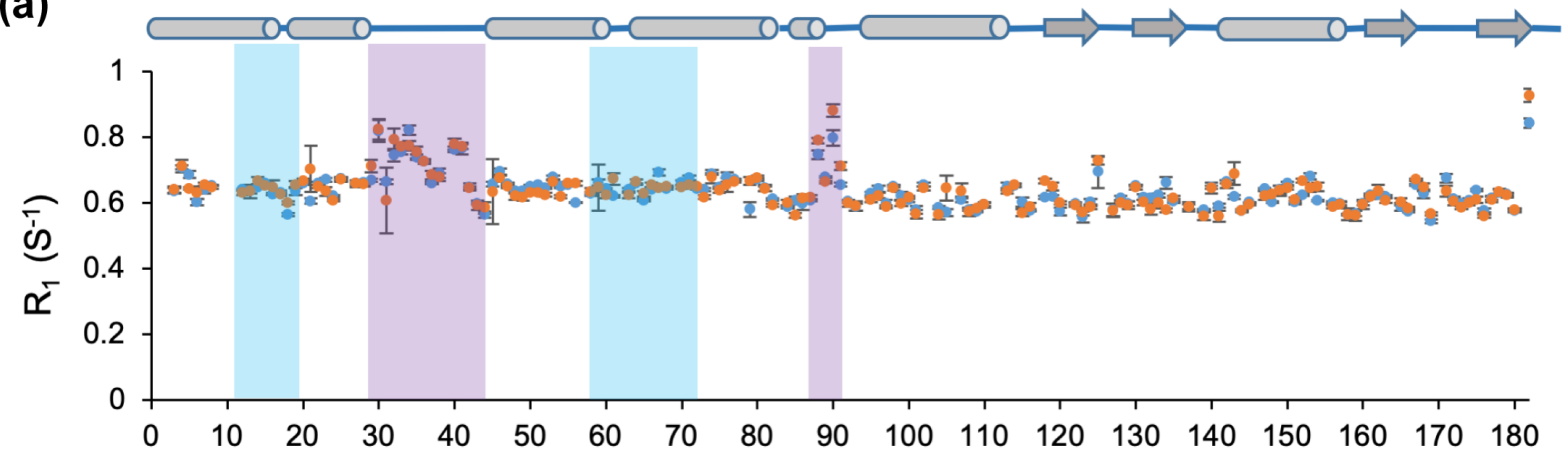

(b)

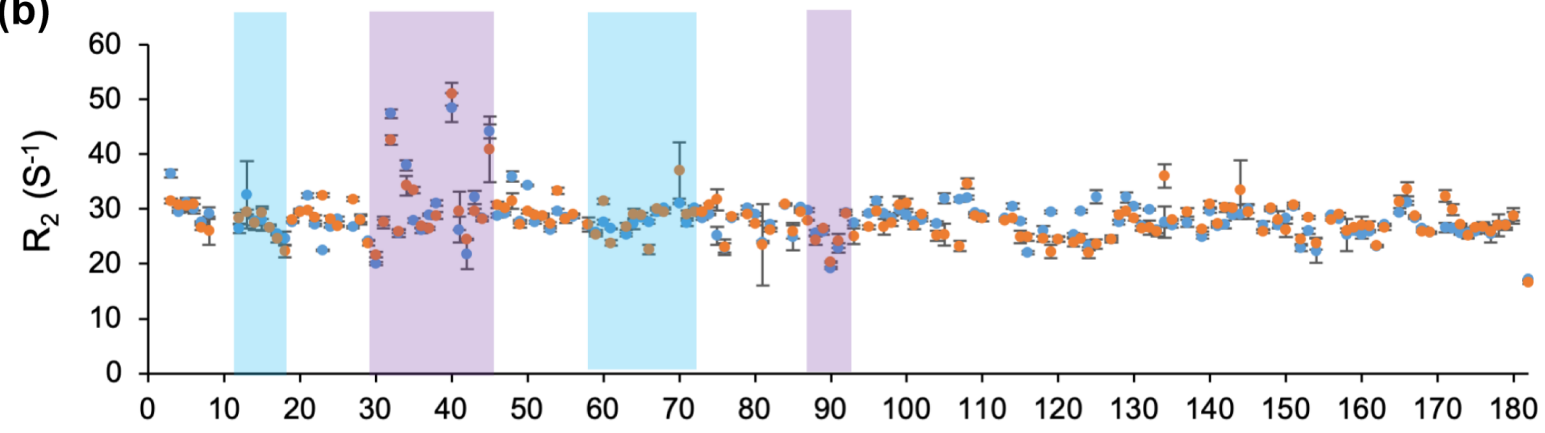

(c)

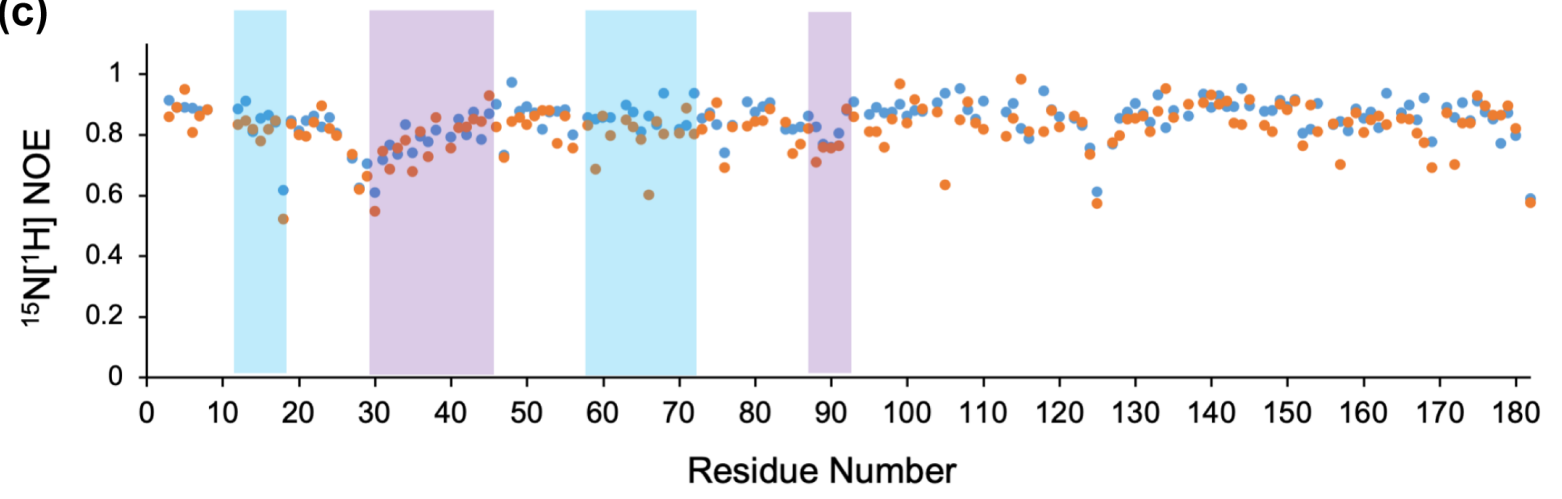

Figure 6 